\title{
PROCESO INNOVADOR Y CAPACIDAD DE CREACIÓN DE ESPACIO DE LOS SERVICIOS AVANZADOS EN ANDALUCÍA*
}

\author{
Rosa Jordá Borrell \\ Departamento de Geografía Física y Análisis Geográfico Regional, \\ Universidad de Sevilla. E-mail borrell@us.es
}

\begin{abstract}
Resumen: El artículo estudia la caracterización del entorno productivo de los servicios avanzados (SA) a través de la creación de espacio relacional. El objetivo es medir su nivel tecnológico y el del espacio productivo andaluz. Se ha investigado sobre el comportamiento tecnológico y no tecnológico de las firmas de SA, las distintas formas de organización de la producción de éstos y los flujos de relaciones (medidos a través de la compra-venta y de la cooperación). Los resultados obtenidos indican que las compañias de servicios avanzados realizan una inversión en $\mathrm{I}+\mathrm{D}$ menor que las empresas industriales, en una proporción de 8 a 1 . Por otra parte, la principal inversión de las empresas de SA es en recursos humanos. Las empresas de gestión de calidad y de consultoría son las que concentran el mayor número de servicios comprados (31,5\% y $31,3 \%$ respectivamente) frente a los Organismos Públicos de Investigación (OPIs) que suministran el grueso de la I+D a los sectores económicos. Gran parte de la contratación de los SA y de la cooperación se realiza a escala regional (63\% y $76,6 \%$ respectivamente) pero la densidad de relaciones es baja y poco continua. Las OPIS y las firmas de gestión de calidad y consultoría se configuran como los elementos clave de la estructuración del espacio innovador andaluz.
\end{abstract}

Palabras Clave: Andalucía, servicios avanzados (SA), comportamiento tecnológico de las firmas de SA, formas de organización de la producción de los SA, sectores demandadores de SA.

\begin{abstract}
This paper studies the features of the environment of production of the Advanced Services (AS) firms in order to understand their technological level. We have also analyzed the environment of production in Andalusia. The investigation has looked for the technological and non-technological behaviour of these firms, organisation of production and the demand flows as regards buying and selling and co-operation relations. The results obtained
\end{abstract}

\footnotetext{
* Recibido: 3-10-2002 Aceptado: 3-5-2003
} 
indicate that the AS companies make a smaller investment on R-D comparing with the investment found in industries, being this in a 8 to 1 proportion. On the other hand, the main investment for the AS companies lies in human resources. Quality control and consulting firms show the highest concentration of services hired $(31.5 \%$ and $31.3 \%$ respectively) in contrast with the Public Research Institutions (PRIs) which provide the largest number of services hired on R-D to the different economical sectors. In most cases, the destination of the services provided by these AS firms and co-operation is found in other companies established in Andalusia ( $63 \%$ and $76.6 \%$ respectively) but the density of relations is low and scarcely continuing. PRIs and quality control and consulting firms are the key elements for the structuration of innovation space in Andalusia.

Key words: Andalusia, Advanced Services (AS), technological behaviour, organisation of production and the demand flows.

\section{Introducción}

A partir de los años ochenta, el crecimiento de los servicios avanzados (SA) ha sido una constante en todas las economías de la OCDE, alcanzando hoy una tasa de un $21 \%$ en la creación de empresas, a la vez que han registrado fuertes incrementos en su ocupación y en la aportación que realizan al Producto Interior Bruto (PIB). En la actualidad, los SA representan en Europa el 12\% del empleo, el $17,2 \%$ del valor añadido (VA) y el $23 \%$ del total de firmas según datos de la Comisión Europea (European Commission, 2001). En España estamos hablando de un $10,5 \%$ en el empleo y un $15 \%$ en el VA de media. Por países tendríamos que las empresas francesas son las que más consumen SA, un 43\%, frente a un $35 \%$ en Alemania, 41\% en Países Bajos y 38\% en Gran Bretaña (European Commission, 2001).

Ahora bien, antes de continuar sería de interés explicar y delimitar brevemente el concepto de servicios avanzados. Se trata de actividades muy especializadas que requieren una alta tecnología y/o una elevada formación del personal ocupado. De ahí que aporten a las empresas clientes un volumen importante de competitividad, productividad e innovación. Por ello, los definimos como un tipo de servicio a empresas de alto contenido en conocimientos que no sólo son innovadores sino que juegan un gran papel en la capacidad innovadora del tejido productivo de las economías desarrolladas (Antonelli, 1999; Miles, 1995). Este hecho da lugar a que sean considerados como estratégicos ${ }^{1}$ para la generación de espacio productivo. 
Su expansión tiene que ver con la necesidad de las sociedades desarrolladas de dar respuesta a los cambios económicos de hoy, tales como los fuertes requerimientos de seguridad y control, el crecimiento de los mercados, el proceso de globalización, la introducción de las nuevas tecnologías y las nuevas formas de organización de las empresas. Transformaciones que a su vez están conectadas con las modificaciones estructurales que se están efectuando en la industria, al estar siendo sustituida o completada por un creciente volumen de servicios. Al mismo tiempo éstos requieren de la inclustria y de las otras actividades económicas para su desarrollo, con lo cual se está generando una terciarización de la producción o de la función productiva de las empresas. Por lo tanto, la configuración de un escenario como éste significa la consolidación y la integración de la economía de los servicios con los demás sectores económicos, especialmente con la industria, dando paso a la sociedad servindustrial o metaindustrial.

Autores como Rayssen (1987), Ochel y Wegner (1987) piensan que la sociedad servindustrial aparece ligada casi exclusivamente al dinamismo innovador de las ramas manufactureras y a aquellas regiones y áreas urbanas que son protagonistas de la expansión de los procesos de globalización. En los países de industrialización tardía con una estructura política descentralizada como España (Jordá Borrell, 2002) uno de los factores explicativos claves del origen y de la expansión de los SA, especialmente en las regiones periféricas (Andalucía, Extremadura, Castilla La Mancha, etc), es la creación del Estado de las Autonomías (1978) y el desarrollo estatutario de éstas. Las investigaciones realizadas en nuestro país correlacionando los procesos de industrialización y el aumento de demanda de SA en las diferentes Comunidades Autónomas arrojan resultados desalentadores (Cuadrado Roura y Rubalcaba, 2000). Parece ser que no existe un patrón claro de relación entre la industria y este sector. Si nos centramos ahora en las regiones periféricas y nos atenemos a las variables globalización y/o mercado único observamos que éstas desempeñan aún hoy un papel secundario, pero incentivador, debido a que tienen una industrialización modesta y una participación en el proceso de internacionalización todavía muy reducido.

El estudio de la actividad empresarial de los servicios avanzados en Andalucía muestra que el $73,4 \%$ de las empresas de este sector se han creado a partir de 1985 y el $84,2 \%$ de ellas desde 1980; pero en verdad, hasta que no se perciben en España las influencias positivas de la entrada en la Unión Europea (UE) a principios de los años noventa, el crecimiento de los SA andaluces aparece ligado sobre todo a las necesidades de la Administración (desarrollo estatutario, nueva ley fiscal, aplicación de las normativas de la UE, construcción de infraestructuras, etc). De forma que ésta en los años 80 y primeros 90 se convirtió en la promotora y principal cliente de las firmas de SA, representando en 1993 el 77\% de su mercado, mientras que en 1997 ya había descendido a un 59\% y hoy ocupa un lugar muy secundario; con ello quere- 
mos poner de manifiesto que los cambios económicos en Andalucía y, en general, en España se han sucedido muy rápidamente.

La puesta en marcha del Plan Andaluz de Desarrollo Económico de 1991-1994 (PADE), las inversiones de la Expo 92' y el mercado único van a desembocar en la expansión de la economía de servicios. Por una parte, porque el PADE 1991-94 supone unos cambios de enfoque respecto a la planificación anterior, poniendo mayor énfasis en la modernización del sistema productivo, en el carácter estratégico del sector industrial, en la estimulación de la iniciativa privada y en la creación y desarrollo de entorno. Por otra, porque la introducción del mercado único implica el inicio de la internacionalización de la economía andaluza que hasta ahora prácticamente no había conocido la apertura al exterior.

Desde comienzos de la década de los noventa, la demanda no pública de SA viene de aquellos sectores que quieren incrementar la cuota de mercado y de los que están introduciendo las nuevas tecnologías y las nuevas formas de organización flexible ${ }^{2}$; para lo cual requieren de servicios de ingeniería y de consultoría a fin de adaptar y mejorar la tecnología existente en el mercado y reciclar la mano de obra. Este proceso supone una aceleración de la expansión de la economía de servicios pasando el número de empresas de 223 en 1993, con un total de 344 establecimientos, a 345 firmas en 1997 y 512 delegaciones/establecimientos (IFA, 1993; 1997). Evidentemente esto es posible porque estábamos en una etapa en la que no existían altas barreras para crear una empresa de servicios avanzados, lo importante era disponer de know how a nivel de adaptación de tecnologías y ser competitivo en la estrategia de reducción de costes ${ }^{3}$. En la actualidad, el total de firmas de SA suman 374 (2001), generan ya el 13,5\% del VAB andaluz (ARDAN, 2001) y en el $45 \%$ de ellas los titulados medios y superiores representan más del $75 \%$ de la plantilla.

\section{Metodología y objetivos}

Dado que los SA en las dos últimas décadas se han convertido en inputs intermedios del proceso productivo e influyen en la competitividad de la empresa (incrementan la calidad, la innovación y la tecnología), el objetivo principal de este trabajo ha sido caracterizar y definir el espacio productivo generado por los servicios avanzados andaluces, a fin de medir el nivel tecnológico de las empresas de servicios y, en consecuencia, de la propia región, utilizando para ello la idea de espacio relacional. Pensamos que a la hora de investigar sobre el fenómeno de la innovación, la noción de continuidad no sirve para explicar su funcionamiento ni las transformaciones producidas por ésta ya que hoy la economía funciona en red y el terri- 
torio a escala regional se fragmenta como resultado de la capacidad competitiva y de organización de las firmas (Camagni, 1991). Se trata, pues, de redescubrir la función del espacio a partir de las relaciones de los proveedores-usuarios de la tecnología y de la innovación en el mundo empresarial. Por consiguiente, hemos considerado a las firmas como elementos clave de la creación del medio innovador andaluz y, en definitiva, como protagonistas de la investigación. De ahí que nos pareció necesario:

1.- Abordar los distintos tipos de modelos de relaciones de las empresas de servicios avanzados, tanto desde el punto de vista innovador como desde la perspectiva del proceso productivo y del mercado. Aspectos estos que nos están empezando a definir las características del entorno productivo andaluz. Ello supone:

a) clasificar los comportamientos tecnológicos de las empresas de servicios avanzados y analizar las estrategias comerciales a través de los diversos modos en que las empresas pueden organizar la obtención y apropiación de recursos técnicos, es decir, desarrollándolos en el interior de las empresas, cooperando con los centros públicos de investigación o con otras empresas; lo que dará lugar a diferentes modelos o regímenes de comportamiento tecnológico entre las firmas (Nelson y Winter, 1984) y a la creación de un medio innovador con una ordenación jerárquica de las actividades tecnológicas en función de las soluciones dadas a los problemas técnicos (radicales, incrementales, imitativas ${ }^{4}$ ). Cuestión esta última que nos puede ayudar a interpretar la estructuración del territorio a diferentes niveles.

b) Estudiar las características de la demanda de los servicios avanzados procedente de los sectores industriales, de la construcción, de los servicios y del sector agrario, al objeto de conocer si se trata de un sistema de relaciones regional y/o extravertido.

c) Analizar las relaciones que establecen las empresas de servicios avanzados con sus proveedores, que en este caso se refiere a la contratación de expertos y de autónomos, así como a la organización espacial de la subcontratación de partes de la elaboración de los servicios avanzados.

d) Como resultado del estudio de los puntos anteriores conocer las características del mercado

Para alcanzar estos objetivos los enfoques que hemos adoptado, tanto para explicar el impacto territorial de las formas de organización relacional como para analizar el proceso innovador de las empresas de SA, han sido el enfoque estruc- 
tural y el sistémico. Dentro del primero, hemos seguido el marco teórico y el método de los evolucionistas. En este marco los rasgos estructurales de las firmas (el tamaño, la capacidad de apertura exterior de la empresa, los medios técnicos, la calidad de los recursos humanos, la experiencia innovadora, etc.) adquieren una importancia primordial para ayudarnos a explicar los regímenes tecnológicos y la capacidad tecnológica e innovadora. De todos ellos, la aplicación del análisis factorial al caso andaluz y español (Jordá Borrell,1998; Buesa y Molero, 1992) ha demostrado que existe una variable, que es la que mejor nos va a ayudar a perfilar el nivel de competitividad empresarial: cuanto más aumenta el tamaño más importancia tiene la cooperación en la creación de recursos tecnológicos y la internacionalización de la empresa (Jordá Borrell, 1997; 1995), lo que favorecerá el potencial innovador y tecnológico.

Por otra parte, las perspectivas teóricas del enfoque sistémico nos permiten interpretar el cambio técnico empresarial de los servicios avanzados en relación con los demás elementos que forman parte del sistema ciencia-tecnología-industria, abarcando las influencias de las instituciones y del mercado. A través de las aportaciones de Porter (1991), de la teoría de la organización de las empresas y de la Geografía de la Organización (Veltz, 1999) cabe insertar las actividades productivas de una firma en dos sistemas interdependientes de redes: a) las de tipo primario o de producción, las cuales constituyen sinergias de proveedores, de empresas de producción, distribuidores, servicios postventa, etc y b) las llamadas actividades de apoyo o servicios avanzados (ingenierías, asesoramiento de $\mathrm{I}+\mathrm{D}$, formación, consultorías, auditorias, etc). Consecuentemente, cuando plasmamos en el territorio esas sinergias podemos conocer su capacidad para construir espacio relacional.

Las nuevas formas de organización de la producción a través del análisis de la oferta-demanda permiten considerar al territorio como matriz de organización y de interacciones sociales, viendo lo que sucede entre los actores de un territorio (entre empresas y entre empresas e instituciones), en la cual la función de los procesos de organización, de comunicación y de cooperación son decisivos desde la perspectiva horizontal y vertical para la expansión de los servicios. En el plano horizontal, porque cuanto mayor es la actividad económica producida por el crecimiento de la demanda de servicios avanzados procedente de los sectores industriales, de la construcción, del agrario, Administración, etc; o cuanto mayor es la demanda generada por el incremento de la cooperación entre el subsistema científico y las empresas en el entorno regional o internacional; cuanta más densidad de relaciones se efectúe, cuanta más creatividad innovadora de carácter endógeno, cuanto mayor es la externalización del proceso productivo en un área, mayor número de sinergias de tipo vertical existirán (subcontratación y contratación de especialistas y expertos), mayor será el proceso de aprendizaje colectivo de carác- 
ter tecnológico y mayor será también el nivel de internacionalización de la economía de la zona estudiada.

Por lo tanto, es evidente que en este sistema van a ejercer un papel estratégico el flujo de información, de conocimiento transferido y la formación. Factores todos que dan lugar a un aumento de la demanda de servicios a empresas y a una jerarquización de los servicios dependiente del nivel tecnológico del tejido productivo, de la internacionalización de la economía, del control del proceso de globalización que ejerce la zona estudiada y del poder económico de las empresas. La eficacia de la organización de todo ese conjunto de empresas e instituciones localiżadas en un lugar o en un área es lo que explica el éxito del proceso innovador del tejido productivo y de la dinámica de los mercados, mucho más que el coste o incluso la calidad de los factores por separado. Por ello, en las investigaciones, y no digamos si son geográficas, resulta fundamental destacar la relevancia de las interacciones científicas, tecnológicas y productivas en el marco regional, tratando de cuantificarlas.

La rapidez con que se está difundiendo la sociedad metaindustrial o serviindustrial hace que se disponga de muy poca información y que no se tengan estadísticas apropiadas para estudiar la problemática de los servicios avanzados. La implantación de estadísticas sobre el proceso innovador de los servicios avanzados se encuentra en proceso de estudio por parte de la Oficina Estadística de la Unión Europea que es la que marca las pautas a seguir a los estados miembros. Hasta ahora sólo ha sido homologado el análisis estadístico del proceso innovador industrial y la creación de la I+D a través del Manual de Oslo y del manual Frascati (OCDE y EUROSTAT, 1994), manuales que permiten la comparación de las estadísticas entre los estados de la UE.

En Andalucía, hasta la fecha se han publicado únicamente dos catálogos de servicios avanzados (IFA, 1993, 1997), en los cuales el número de variables que aparecen es muy reducido y como cabe deducir de las fechas de publicación apenas podemos estudiar el proceso de expansión de los SA en Andalucía. Dada la escasez de información que ofrecían éstos, optamos por crear un Directorio con la información de dichos catálogos y las empresas nuevas que hemos localizado en bases de datos existentes en internet (Parque Tecnológico de Málaga, Andalucía Innova, Ardan, CDTI, o por contactos establecidos con organismos andaluces). Por otra parte, teniendo en cuenta el escaso número de variables que nos ofrecían estas bases de datos (localizadas en internet y en los catálogos consultados), decidimos entrevistar a las empresas andaluzas de SA mediante un amplio cuestionario de unas 600 variables que cubrieran los campos que vamos a desarrollar en este artículo. No obstante quisiéramos advertir que la investiga- 
ción de la cual hemos partido, forma parte de un proyecto más amplio, desarrollado a partir de la elaboración de tres cuestionarios distintos, uno para servicios avanzados, otro para las empresas industriales y otro para los servicios en general y la construcción, si bien en esta ocasión hemos utilizado fundamentalmente los resultados del trabajo de campo concerniente a los establecimientos de servicios avanzados y de industria.

Para llevar a cabo el tratamiento estadístico de los tres conjuntos de sectores estudiados mantuvimos la estructura y el contenido de los cuestionarios de forma semejante, aunque somos conscientes de las diferencias que existen en los procesos de producción y en los de innovación de las actividades analizadas. En principio seleccionamos una muestra de 230 elementos mediante un muestreo sistemático estratificado por sectores, tamaño y localización provincial. De ese total obtuvimos una entrevista-encuesta completa de 210 , de las que 75 correspondían a establecimientos de SA, 75 a industrias y el resto a servicios en general y construcción. Las entrevistas se efectuaron a lo largo del año 2001, habiendo comprobado con las técnicas de muestreo que se suelen emplear en estos casos que la muestra es representativa de la población de las firmas existentes en la actualidad.

\section{Proceso de Innovación de los servicios avanzados andaluces}

El interés de adaptar algunos de los modelos teóricos del proceso innovador a la realidad andaluza obedece a que cada uno de ellos hace hincapié en alguna variable clave de la innovación productiva, pudiendo estudiar esta última desde una perspectiva regional y sistémica evolutiva, al objeto de: 1.- conocer las características del espacio relacional construido y 2.- destacar la importancia de la ordenación jerárquica de las actividades tecnológicas, según las soluciones dadas por las empresas a los problemas técnicos (radicales, incrementales, imitativas). Por tanto, la territorialidad resultante es una consecuencia de la capacidad organizativa e innovadora de las firmas del medio innovador; lo que nos lleva a pensar que para conocer la magnitud del espacio creado, previamente debemos profundizar en el papel jugado por las empresas.

Así, el modelo de Kline y Rosemberg (1986) está basado en las ideas: a) la investigación es un medio fundamental de acceder a la tecnología y b) el proceso innovador se genera al desarrollarse el sistema ciencia-tecnología-industria. Ciertamente, podría encajar con el comportamiento tecnológico de un grupo de 
empresas andaluzas; si bien, para ello se tendría que modificar el modelo en algunas características dado que las actividades de $\mathrm{I}+\mathrm{D}$ realizadas por las firmas establecidas en la región son mayoritariamente de desarrollo tecnológico (80,3\% de los gastos) y en menor medida de investigación aplicada $(18,9 \%)$, mientras que la básica apenas supone el $0,8 \%$ del total de gastos. Hecho que explica la poca participación de estas empresas en programas de I+D europeos y el que los créditos concedidos por el CDTI (Centro de Desarrollo Tecnológico Industrial de carácter estatal) vayan dirigidos sustancialmente hacia proyectos de desarrollo tecnológico.

Por otra parte, existen otros grupos de empresas cuyo comportamiento tecnológico estaría mejor explicado por el modelo de Marquis (1969) mejorado por Utterback (1971), pues, si estudiamos los motivos que impulsan a muchos empresarios andaluces de servicios avanzados a innovar, vemos que los objetivos más valorados de la actividad innovadora son mejorar la calidad de los servicios existentes, aumentar la cuota de mercado en España o en el extranjero y reducir costes. Sin embargo, no se encuentran entre sus metas crear nuevos métodos o metodologías para desarrollar los servicios existentes, generar nuevos servicios, investigar mediante proyectos para ver como mejorarlos, etc. Luego entonces nos estamos refiriendo a empresas que tienen como finalidad elaborar servicios mejorados mediante la práctica diaria (innovación incremental) o bien que se ven forzadas a crearlos por causas externas a la firma (publicación de una nueva legislación, aparición de nuevos competidores, de productos sustitutivos, etc). En este contexto, sería conveniente considerar la innovación como una manera de solucionar problemas, no como una vía para crear un producto o servicio totalmente nuevo, ni como una forma de hacer un descubrimiento. En las empresas que innovan así, la idea inicial de creación del producto o servicio no procede del departamento de investigación ni es resultado de la realización de $\mathrm{I}+\mathrm{D}$ (generalmente no existe).

De acuerdo con los resultados de nuestro trabajo podemos diferenciar tres subgrupos de empresas según su comportamiento innovador, los cuales vamos analizar a continuación y que serían:

A. Las empresas que realizan innovaciones por medio de I+D.

B. Las que llevan a cabo innovaciones tecnológicas sin desarrollar I+D y por último

C. Aquéllas que innovan a través de innovaciones no tecnológicas, introduciendo mejoras de organización, creación de know how, etc. 


\section{Tipos de comportamientos tecnológicos de las empresas de servicios avanzados}

A Para analizar al conjunto de empresas que llevan a cabo I+D hemos utilizado tal como anunciamos antes el modelo de Kline y Rosemberg adaptado. Aún así, los resultados de la investigación ponen de manifiesto que estas empresas andaluzas se ajustan con dificultad a dicho modelo porque:

1.- Se trata sobre todo de empresas pertenecientes a la consultoría $(29,6 \%$ del sector), ingeniería (ciertos tipos de ingeniería como medio ambiente, energía y bienes de equipo: $42,1 \%)$, diseño $(6,5 \%)$, laboratorios de I+D $(10,9 \%)$ e informática $(10,9 \%$ del sector), que representan el 36\% de las firmas de servicios avanzados y que utilizan el $47 \%$ de los gastos totales de innovación tecnológica (para el desarrollo de metodologías y de técnicas nuevas, creación de herramientas informáticas, etc) o el $22,8 \%$ de la innovación tecnológica y no tecnológica, pero que no siempre hacen $\mathrm{I}+\mathrm{D}$ de forma sistemática ${ }^{5}$. Es necesario, por tanto, comprender que la innovación de estas empresas surge del contacto con la ciencia a lo largo de todo el proceso, bien utilizando como fuente las publicaciones (en este caso recurriendo a soluciones ya existentes), bien buscando respuestas a los problemas en el desarrollo de I+D. Pero cuando estas firmas efectúan investigación y desarrollo, la financiación procede de las propias empresas en un $7 \%$ y el resto corresponde a fondos ajenos pertenecientes a la administración pública, lo cual nos está indicando que aún no creen suficientemente en la I+D como ventaja competitiva.

De la misma manera que todavía no han llegado a ver como beneficio la utilización de los acuerdos de cooperación, por ejemplo la alianza estratégica para reducir costes y aumentar las economías de escala; puesto que muy pocas empresas efectúan la $I+D$ en cooperación (17\%) y sus gastos representa el $11,2 \%$ del total de los de innovación. Si bien, cuando se coopera se lleva a cabo teniendo como socio a las universidades andaluzas, ya que la colaboración con el CSIC y con otras empresas no tiene relevancia (2\%). El ámbito de la cooperación científica corresponde a la escala regional fundamentalmente y cuando se lleva a cabo ésta se realiza dentro de la misma ciudad concentrándose sobre todo en Sevilla, Almería, Huelva y Málaga. Por lo tanto, empezamos a tener, por un lado, pequeños espacios islas, urbanos a nivel sobre todo de capitales de provincia, generados a partir de la cooperación de I+D y, por otro, puntos no enlazados que corresponden a firmas o delegaciones que no favorecen el desarrollo del sistema ciencia-tecnologíaindustria (no cooperan) y que se localizan igualmente en dichas capitales.

2.- Si anteriormente hemos dicho que la innovación surge de la sinergia ciencia-tecnología-industria a lo largo de todo el proceso innovador, ahora 
correspondería analizar las relaciones de este grupo de empresas con el mercado de proveedores:

a) Viendo cómo incide la compra de tecnología en el proceso innovador, pues el porcentaje de empresas que manifiestan adquirir tecnología desincorporada es muy escaso, tan solo el 12,12\%. Además, ésta procede de agentes nacionales y andaluces más que de firmas extranjeras, excepto en el caso de las marcas. Las razones que cabe aducir sobre este hecho serian que, salvo una minoría que realizan I+D desde principios de los años 80 , la mayoría de las firmas andaluzas vienen acometiéndolo desde principios de 1990 (Figura 1), lo cual quiere decir que tienen poca experiencia o acumulación de conocimientos. Evidentemente, en estas circunstancias, la oferta tecnológica de los agentes nacionales se adapta mejor a las necesidades específicas de las empresas andaluzas, lo que nos está corroborando una vez más la escasa madurez tecnológica de las empresas de servicios avanzados y su débil presencia en los mercados internacionales desde el punto de vista tecnológico, dado el escaso peso de la importación de tecnología. Por lo tanto, el otro brazo de la modernización tecnológica de este grupo de firmas corresponde a la compra de bienes de equipo informáticos, programas y sobre todo asistencia técnica, reciclaje y formación continua.

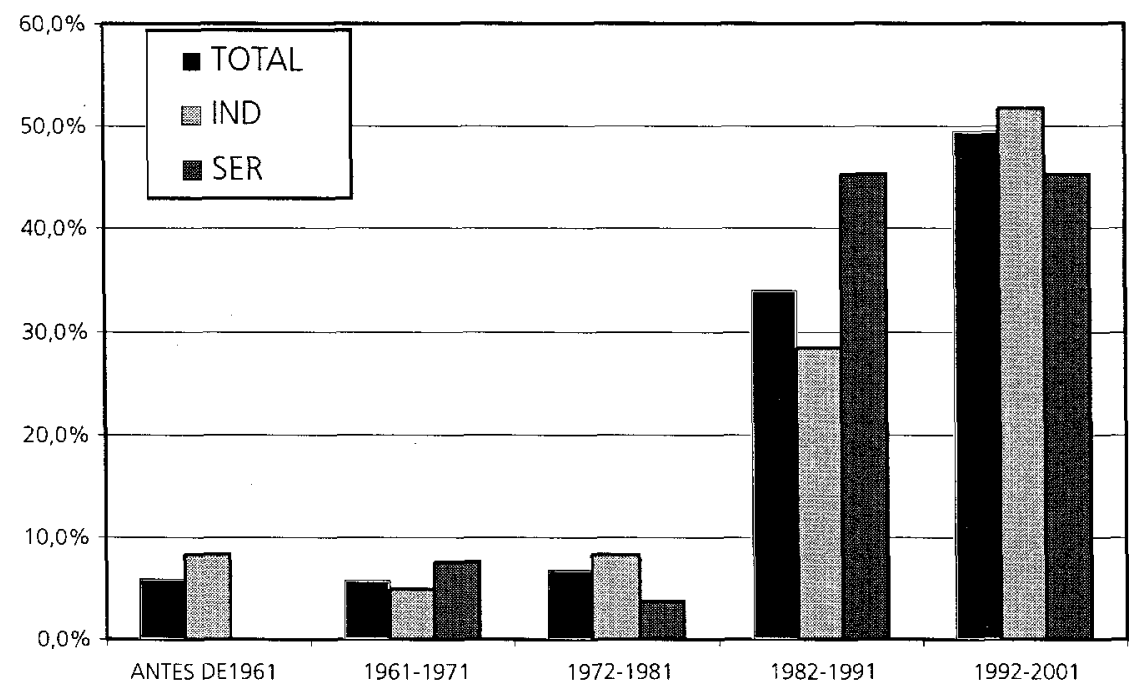

Figura 1. Distribución porcentual de las empresas encuestadas según año de comienzo de las actividades económicas. (Fuente: Elaboración propia a partir de nuestras encuestas) 
b) Estudiando la influencia en el proceso innovador de la contratación de autónomos. Pues estas empresas, como no generan productos repetidos, sino personalizados, para reducir costes y poder disponer de la diversidad de especialistas que requieren en cada momento, contratan temporalmente a autónomos y a expertos independientes aumentando así la competitividad y la diferenciación del producto. Estos especialistas que representan en el proceso de elaboración del servicio el $10 \%$ del coste total, proceden en un $60 \%$ del entorno regional o provincial, en un 30\% del resto de España y un $10 \%$ se buscan en el extranjero. De ello deducimos que el ámbito de contratación de los especialistas es sobre todo regional, generándose mercados fraccionados de carácter provincial, cuyo centro es la capital.

3.- Una vez vistas las características de los inputs para llevar a cabo el proceso innovador, vamos a analizar el alcance de los resultados de dicho proceso, es decir, la magnitud del mercado. El mercado de estas empresas que hacen $\mathrm{I}+\mathrm{D}$ es nacional y extranjero en un $29 \%$, incorporando innovaciones estratégicas para desarrollar proyectos y contratos con empresas de otros países de la UE, norte de África, América Latina y USA a través de la creación de uniones temporales de empresas (UTEs), que representa el 10\% de las ventas. En el mercado español, compran acciones (un 18\% del total empresas) de otras firmas para poder ejercer en esas Comunidades Autónomas y de esa forma extender la gama de servicios y aumentar la cuota de ventas en nuestro país.

Por lo tanto, estas empresas tienen como objetivo incrementar el mercado asociándose estratégicamente con otras firmas del mismo sector (65\% del total), aumentar las economías de escala (55\%) y mejorar de forma continua sus servicios a través de la transferencia de conocimientos que se produce al asociarse o al practicar el outsourcing a través de empresas andaluzas que se establecen en el mercado español ( $50 \%$ del total de firmas) y extranjero. Desde el punto de vista geográfico lo que llevan a cabo estas firmas es buscar socios del mismo nivel tecnológico o innovador a distancia poniendo en marcha las ventajas del vecinaje tecnológico (Fache, 2002). Generalmente esta estrategia se adopta cuando las ventas se desarrollan en Europa y USA (25\%), mientras que con los países subdesarrollados (32\% del total de firmas) la forma de colaboración más utilizada es la contratación pública o concurso internacional para aprovecharse de la baratura de la mano de obra y vender allí los nuevos servicios o el know how, caso de la ingeniería.

Pero la cuestión más importante a destacar es que la venta de servicios nuevos se hace en un $90 \%$ en Andalucía mediante la forma de asistencias téc- 
nicas, entre ellas la práctica del outsourcing, de licencias de software y de plantas llave en mano que comercializan a la agroindustria, papel, construcción de maquinaria, material de transporte y en menor medida a la industria química, materias plásticas y de comercio; representando esta venta de tecnología y know how desincorporado el $45 \%$ de la facturación. En este escenario, la escala de los mercados es sobre todo de carácter local, provincial o metropolitano (Figura 2).

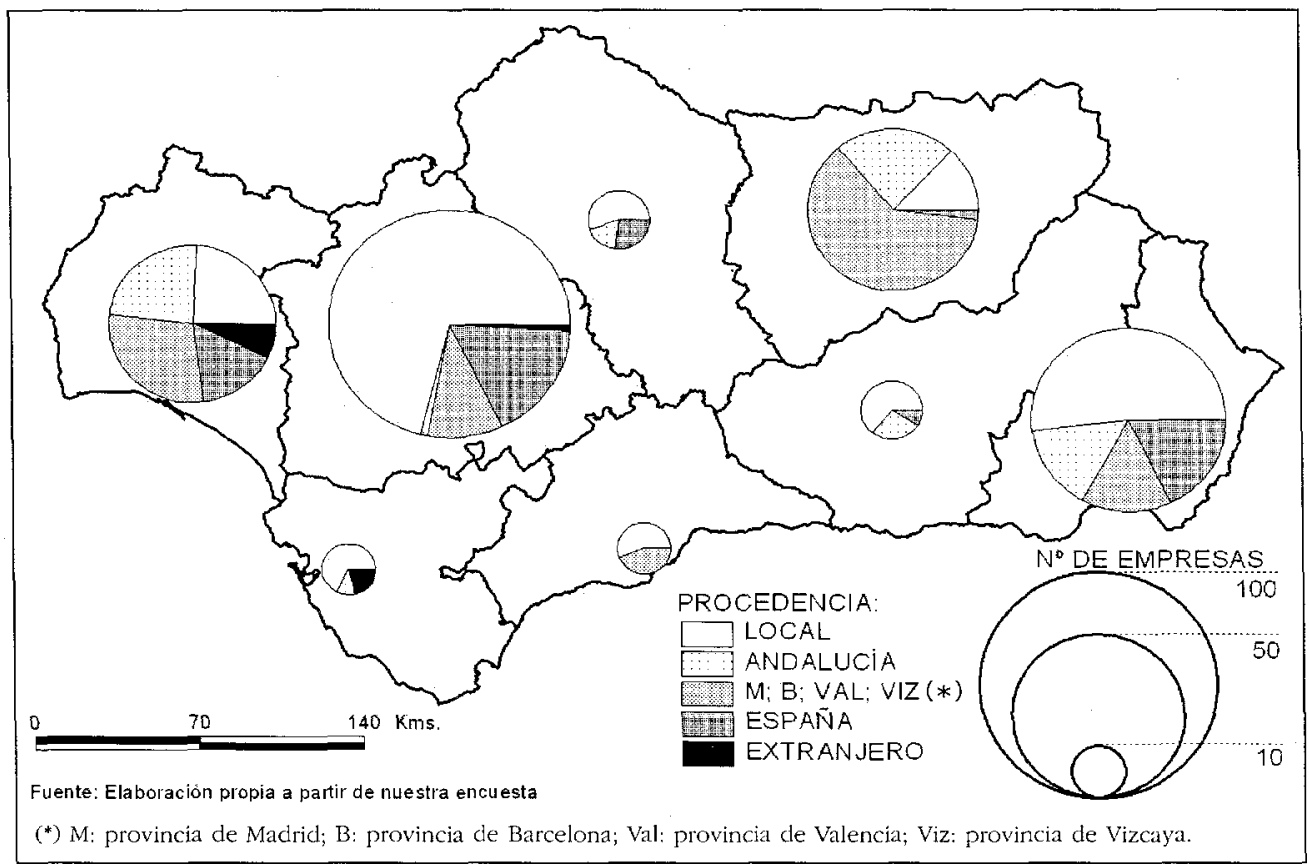

Figura 2. Empresas de servicios avanzados según su localización contratadas por empresas industriales

En resumen, las variables clave que definen este tipo de servicios avanzados serían: realización de $\mathrm{I}+\mathrm{D}$ no siempre sistemático, obtención de innovaciones incrementales (nunca radicales) formadas por mejoras continuas de los servicios ya conocidos, escasa importación de la tecnología, comienzos de internacionalización en la venta y elaboración de servicios, sistemas de relaciones fundamentalmente locales/provinciales (40\%), regionales (17\%) en el ámbito comercial y en el transcurso de la elaboración del producto.

B El $48 \%$ de las empresas de servicios avanzados que realizan innovaciones tecnológicas de forma sistemática sin llevar a cabo I+D se ajustan con mayor exactitud al modelo de Marquis y Utterback descrito anteriormente porque: 
1.- Este grupo de empresas a la hora de innovar parten de una idea sobre una mejora de producto o método o técnica que han visto a la competencia, que les ha aportado un cliente o que han aprendido de la multinacional donde trabajaban anteriormente, consistiendo la innovación en hallar una combinación nueva de tecnologías existentes. Por ello, esa idea surge para solucionar un problema o una necesidad a los clientes.

Y después, en función de la envergadura de esa dificultad a resolver, se asocian o no con otras empresas, desarrollan o no proyectos, subcontratan parte del proceso o contratan a expertos y/o especialistas. De ahí que la cooperación con otras firmas, sobre todo con las de clientes, represente el $60 \%$ del total de la asociación que hace el grupo, mientras que con las OPIs (Universidad, CSIC) es mucho menos significativa, el 25\% (principalmente se contrata $\mathrm{I}+\mathrm{D}$ ), y el resto corresponde a la contratación de autónomos y expertos. Merece la pena destacar en este grupo la práctica de algunas empresas medianas y grandes que no tienen experiencia en realizar I+D y se asocian con multinacionales de otro subsector de SA establecidas en Andalucía (generalmente de informática o internet) para desarrollar nuevos servicios o mejorarlos, teniendo a ésta última como socio tecnológico. Ésta es una práctica habitual entre las ingenierías, las informáticas y las consultoras.

El sistema relacional generado es a primera vista de ámbito regional, pero, dado que priman las interrelaciones de proximidad, la organización resultante sigue estando ligada a un espacio local/provincial. En este sentido, podemos afirmar que el $52 \%$ de los flujos de este grupo empiezan y terminan en cada una de las áreas de influencia de las capitales siguientes: Sevilla, Almería, Huelva y Jaén. Y por ello cabe hablar igualmente de construcción de espacios fragmentados.

Por otra parte, los empresarios de este grupo están más dispuestos a gastar fondos suyos en innovación que en $\mathrm{I}+\mathrm{D}$, ya que la financiación propia representa un 30\%. Además, el número total de proyectos realizados y subvencionados en los últimos cinco años por el CDTI y el Plan Nacional de innovación es superior a los desarrollados por el grupo de empresas que hacen $\mathrm{I}+\mathrm{D}$. Incluso el montante de los gastos de innovación efectuados por este grupo supone el 53\% del total de los llevados a cabo por todas las empresas andaluzas de servicios avanzados. De forma que también es superior a la suma gastada por las firmas de I+D. La diferencia de comportamiento fundamental de estas empresas de innovación respecto de las de $\mathrm{I}+\mathrm{D}$, además del volumen de recursos gastados estriba: 
a) en la secuencia seguida para lograr la innovación o desarrollar el proceso innovador de acuerdo con el modelo lineal. Pues, al no requerir investigación básica o aplicada estas innovaciones, los tipos de proyectos más destacados son los de ingeniería, diseño y adaptación, con lo cual la secuencia seguida para lograr la innovación es más corta y menos intensa en términos tecnológicos; por lo tanto la capacidad de estas firmas para incrementar el nivel tecnológico regional es más reducida. Ahora bien, como la cooperación con empresas para este grupo de firmas es importante, la densidad de la malla o del flujo es mucho mayor, favoreciendo la difusión del conocimiento a niveles tecnológicos mas bajos que la $\mathrm{I}+\mathrm{D}$. Además, estas empresas hacen innovación sistemática, disponen de un número fijo de técnicos superiores y medios para desarrollar esos proyectos, situándose la media en 6,6 y la innovación en cooperación representa el $39 \%$ del total de gastos de innovación. Sin embargo, las empresas que realizan $\mathrm{I}+\mathrm{D}$ no siempre disponen de técnicos medios o superiores con un carácter fijo dedicados a ese menester.

b) La otra cuestión a destacar del comportamiento tecnológico de este conjunto de empresas es que producen fundamentalmente servicios mejorados (60\%) y exportan muy poco (2\%) dedicándose sobre todo al mercado regional y nacional, estando constituido el grupo por todos los subsectores de servicios avanzados de ingenierías, consultoras, gestión de calidad, informática, laboratorios de pruebas y ensayo, salvo publicidad, diseño y los laboratorios de I+D.

C Por último, existen un 16\% de las empresas de SA correspondientes a los subsectores de consultoras jurídicas, auditorías, las especializadas en gestión (planificación estratégica, asesoramiento económico, control de gestión, estudios de mercado), o las de publicidad que no siempre crean o mejoran tecnología para innovar. Estos pueden llegar a vender nuevos servicios o servicios mejorados utilizando y adaptando las nuevas tecnologías de la información en el desarrollo de sus procesos, invirtiendo en formación, en innovaciones no tecnológicas, es decir, mejorando su organización e introduciendo estrategias de mercado y generando know how propio; por lo tanto estas empresas podrían hacer algún tipo de investigación.

Se trata, pues, de un grupo de empresas en el que al menos cabe diferenciar dos subconjuntos: aquéllas que utilizan técnicas y métodos normalizados y reglamentados, por ejemplo, las empresas de gestión de calidad, control y 
seguridad, y las auditorías, en las cuales, lógicamente no puede haber creación ni innovación, a no ser que sean de tipo organizativo o estratégico o que incorporen la utilización de aparatos complejos. Y, en segundo lugar, tendríamos el conjunto constituido por las consultoras especializadas en gestión y mercado (salvo las auditoras) y las empresas de publicidad, en un 58\% de las cuales se han incorporado modificaciones en la organización interna de las firmas a través de las transformaciones operadas en la división funcional del trabajo y en las tareas clave.

Los avances de estas empresas, que son pequeñas y medianas empresas (PYMEs) de hasta 2,5 millones de euros de facturación, salvo las de gestión de calidad que se corresponden con multinacionales, obedecen más a factores externos (cambios de la legislación, cambios informáticos) que a la política interna, por ejemplo, a la introducción de la certificación de calidad (un 45\% de ellas poseen la certificación y han adaptado modernas técnicas de gestión). $\mathrm{Y}$ las fuentes para mejorar y crear nuevos productos son los servicios prestados a los clientes, conferencias, publicaciones y el grado de amenaza que perciben por la entrada de nuevos competidores o servicios sustitutivos.

En consecuencia, estas empresas adoptan fundamentalmente la estrategia mixta de reducción de costes y la maximización de la calidad y eficacia de resultados más que la innovación tecnológica. De ahí que el factor clave que clasifica a estas firmas como verdaderamente innovadoras es el rango del mercado que logran alcanzar, es decir, la conquista de una cuota de mercado nacional o internacional para sus servicios frente al regional. Sin embargo, la mayoría de ellas tienen un mercado cien por cien regional y local, salvo un $29,6 \%$ de consultoras que hacen I+D y un $8 \%$ de las firmas de publicidad y de gestión de calidad que realizan innovación tecnológica vendiendo al mercado nacional e internacional un $30 \%$ de su producción de servicios, de las cuales ya hemos hablado puesto que pertenecen a los grupos A y $\mathrm{B}$, respectivamente.

Por consiguiente, desde el punto de vista de la innovación tecnológica, este grupo (C) apenas diseña un sistema ciencia-tecnología, debido a que la introducción de las innovaciones tecnológicas en la empresa es ocasional, generalmente impulsada desde fuera, y porque las conexiones con el entorno científico y tecnológico prácticamente no se dan, salvo en el caso de algún contrato de formación. Se localizan exclusivamente en cada una de las ocho capitales andaluzas más Jerez y venden los servicios/productos nuevos en el mercado andaluz con un carácter local/provincial, pero evidentemente no registran el know how y el servicio nuevo ni en España ni en el extranjero, es decir, se trata de un producto novedoso para el mercado de la propia empresa o a escala local/provincial, que incluso cabría calificar como innovación imitativa. 


\section{Relaciones de los Servicios Avanzados con los sectores económicos andaluces}

Teniendo en cuenta que las características de las relaciones de los servicios avanzados con los sectores económicos están estrechamente vinculadas con los rasgos del proceso innovador andaluz, vamos a estudiar estos flujos, desde dos perspectivas complementarias:

1.- A partir del número de contratos establecidos y el porcentaje de compras entre las empresas de servicios avanzados y el resto de las firmas pertenecientes a las demás ramas económicas, según información suministrada por las empresas de servicios avanzados (Tabla 1). Información que, a su vez, hemos contrastado con la ofrecida por las empresas de las actividades agrarias, construcción, servicios, industria y administración (Tabla 2). De esta forma podremos conocer no sólo la importancia de la demanda regional de los servicios avanzados sino también el porcentaje de estos servicios que se contratan fuera de Andalucía y sus causas.

Tabla 1. Compra de servicios avanzados por parte de las empresas innovadoras industriales por sectores (\% en horizontal)

\begin{tabular}{|c|c|c|c|c|c|c|c|c|c|c|c|c|c|c|c|}
\hline \multirow{3}{*}{ Servicio comprado } & \multicolumn{13}{|c|}{ Sector comprador $(x)$} & \multicolumn{2}{|c|}{ Comratados en: } \\
\hline & $A$ & $\overline{D A}$ & $D E$ & $\overline{D G}$ & $\overline{D H}$ & $D I$ & $D J$ & $\overrightarrow{D K}$ & $D L$ & & $D N$ & $\bar{G} T$ & tal & & \\
\hline & & & & & & & & & & & & & & NDAL. & SPANA \\
\hline Selección y evaluación de recursos humanos & 0 & 1,7 & 0,4 & 0,4 & 0,4 & 0,4 & 09 & 0,4 & 0 & 0.4 & 0,4 & 0.9 & 6,4 & 87 & 13 \\
\hline Formación & 0 & 2,6 & 0.9 & 0,4 & 0,4 & 0,9 & 0,9 & 0,9 & 0 & 0,9 & 0 & 0,9 & 8,5 & 80 & 20 \\
\hline Métodos y tiempo & 0 & 0,4 & 0 & 0 & 0.4 & 0 & 0 & 0 & 0 & 0,4 & 0 & 0 & 1,3 & 67 & 33 \\
\hline Estudio de costes & 0 & 0 & 0 & 0 & 0.4 & 0 & 0 & 0 & 0 & 0,4 & 0 & 0,4 & 1,3 & 0 & 100 \\
\hline Diseño industrial & 0 & 1,3 & 0 & 0,4 & 0,4 & 0 & 0 & 0,4 & 0 & 1,3 & 0 & 0 & 3,8 & 44 & 56 \\
\hline Ingeniería de bienes & 0 & 0,9 & 0 & 1,3 & 0 & 0 & 0,4 & 0 & 0 & 0 & 0 & 0 & 2,6 & 50 & 50 \\
\hline Gestión de calidad & 0,4 & 1,7 & 0,4 & 0,9 & 0,9 & 0,4 & 1,3 & 2,6 & 1,3 & 0,4 & 0,4 & 0,9 & 11,5 & 74 & 26 \\
\hline Asesor: & 0 & 0 & 0 & 0,4 & 0 & 0 & 0 & 0,4 & 0 & 0 & 0,4 & 0,4 & 1,7 & 25 & 75 \\
\hline Imagen y publicidad & 0,4 & 2,6 & 0,4 & 1,3 & 0,9 & 0,4 & 0 & 0,4 & 0,9 & 0,9 & 0 & 0,4 & 8,5 & 60 & 40 \\
\hline Diseño gráfico & 0,4 & 1,7 & 0 & 0,4 & 0 & 0,4 & 0 & 0,4 & 0,4 & 0,4 & 0 & 0 & 4,3 & 60 & 40 \\
\hline Estudio de mercado & 0 & 0,9 & 0 & 0,9 & 0 & 0 & 0 & 0 & 0,4 & 0,4 & 0 & 0,9 & 3,4 & 38 & 63 \\
\hline Asesoramiento comercial & 0 & 0,9 & 0 & 0 & 0 & 0 & 0 & 0 & 0 & 0 & 0 & 0.4 & 1,3 & 33 & 67 \\
\hline logístico & 0 & 1,3 & 0 & 0 & 0 & 0 & 0 & 0,4 & 0 & 0 & 0 & 0,4 & 2,1 & 40 & 60 \\
\hline Organización de empresas & 0 & 1,3 & 0 & 0 & 0,4 & 0 & 0 & 0,4 & 0,4 & 0,9 & 0,4 & 0,9 & 4,7 & 27 & 73 \\
\hline Asesoramiento económico & 0 & 1,3 & 0,4 & 0 & 0,4 & 0 & 0 & 1,7 & 0,4 & 0 & 0,4 & 0,4 & 5,1 & 58 & 42 \\
\hline Control y gestión & 0 & 0,9 & 0 & 0 & 0 & 0 & 0 & 0,9 & 0 & 0 & 0 & 0 & 1,7 & 75 & 25 \\
\hline ón estratégica & 0 & 0,9 & 0 & 0 & 0 & 0 & 0 & 0 & 0 & 0 & 0 & 0 & 0,9 & 50 & 50 \\
\hline Implantación sistemas informáticos & 0,9 & 2,1 & 0,4 & 0,9 & 0,9 & 0 & 0,4 & 0,9 & 0 & 0,4 & 0 & 0,4 & 7,2 & 82 & 18 \\
\hline Programas informáticos & 0,4 & 2,1 & 0,4 & 0,9 & 0,4 & 0 & 0 & 0,4 & 0 & 1,3 & 0 & 0,4 & 6,4 & 73 & 27 \\
\hline Implantación telecomunicaciones & 0 & 1,3 & 0,4 & 0 & 0 & 0,4 & 0 & 0,4 & 0 & 0,9 & 0 & 0 & 3,4 & 75 & 25 \\
\hline Centro de cálculo & 0 & 0,4 & 0 & 0 & 0 & 0 & 0 & 0 & 0 & 0 & 0 & 0 & 0,4 & 100 & 0 \\
\hline Localización de plantas & 0 & 0 & 0 & 0 & 0 & 0 & 0 & 0 & 0,4 & 0 & 0 & 0 & 0,4 & 100 & 0 \\
\hline Impacto ambiental & 0 & 1,7 & 0,4 & 1,7 & 0 & 0,4 & 0,9 & 0 & 0 & 0,4 & 0 & 0,4 & 6 & 71 & 29 \\
\hline Ingeniería civil & 0 & 1,7 & 0 & 0,9 & 0 & 0,4 & 0,4 & 0,4 & 0 & 0 & 0 & 0,4 & 4,3 & 50 & 50 \\
\hline Inversión y estudios viabilidad & 0 & 1,3 & 0 & 0,4 & 0 & 0 & 0 & 0 & 0 & 0,4 & 0 & 0 & 2,1 & 40 & 60 \\
\hline Estudios socioeconómicos & 0 & 0,4 & 0 & 0,4 & 0 & 0 & 0 & 0 & 0 & 0 & 0 & 0 & 0,9 & 100 & 0 \\
\hline $\mathrm{N}^{\circ}$ CONTRATOS/ EMPRESA/AÑO & 1,5 & 7,3 & 5 & 3 & & 2,25 & 2 & 2,20 & 0,77 & 4,6 & 1 & 10 & 3 & & \\
\hline TOTAL & 2,6 & 31,1 & 4,3 & 11,5 & 6 & 3,8 & 5,1 & 11,1 & 4,3 & 9,8 & 2,1 & 8,5 & 100 & 63 & 37 \\
\hline
\end{tabular}

Fuente: Elaboración propia a partir de nuestras encuestas.

(x) Véase clave en Anexo I 
Tabla 2. Contratos de las empresas de servicios avanzados según sectores de clientes (\% en horizontal)

\begin{tabular}{|c|c|c|c|c|c|c|c|c|c|c|}
\hline \multirow[b]{2}{*}{ Sector Cliente } & \multicolumn{10}{|c|}{ Servicio contratado $(\mathrm{x})$} \\
\hline & Cal & Con & Dis & Infor & Ing & Intent & Labor & $\begin{array}{l}\text { Labor } \\
I+D\end{array}$ & Public & $\begin{array}{l}\text { \% del } \\
\text { Tolal }\end{array}$ \\
\hline Administración & 14,7 & 31,7 & 2,4 & 9,8 & 17,1 & 2,5 & 2,4 & 7,3 & 12,1 & 11,5 \\
\hline Ocio y turismo & 16,7 & 33,3 & 5,6 & 11,1 & 5,6 & 5,6 & 11,1 & 0 & 11 & 4,9 \\
\hline Comercio & 18,8 & 43,6 & 0 & 12,5 & 6,3 & 0 & 6,3 & 0 & 12,5 & 4,3 \\
\hline Hostelería & 25 & 37,5 & 0 & 6,3 & 6,3 & 0 & 12,4 & 0 & 12,5 & 4,3 \\
\hline Comunicaciones & 23,1 & 30,7 & 7,7 & 15,4 & 7,7 & 0 & 7,7 & 0 & 7,7 & 3,5 \\
\hline Transporte & 33,3 & 50 & 0 & 0 & 8,3 & 0 & 8,4 & 0 & 0 & 3,2 \\
\hline Financieros & 25 & 33,3 & 8,3 & 16,7 & 0 & 0 & 0 & 0 & 16,7 & 3,2 \\
\hline Sanidad & 33,3 & 33,3 & 0 & 8,3 & 8,3 & 0 & 0 & 8,3 & 8,5 & 3,2 \\
\hline Agua & 36,4 & 36,4 & 9,1 & 0 & 18,1 & 0 & 0 & 0 & 0 & 3 \\
\hline Ingeniería & 28,6 & 21,4 & 0 & 14,4 & 21,4 & 0 & 7,1 & 7,1 & 0 & 3,8 \\
\hline Otros Servicios & 50 & 33,3 & 0 & 0 & 16,7 & 0 & 0 & 0 & 0 & 1,6 \\
\hline Construcción & 25 & 50 & 12,5 & 12,5 & 0 & 0 & 0 & 0 & 0 & 2,2 \\
\hline Agrario & 23,1 & 46,2 & 0 & 7,7 & 7,7 & 0 & 7,7 & 0 & 7,7 & 3,5 \\
\hline Industria agroalimentaria & 20 & 33,3 & 6,7 & 13,3 & 13,3 & 0 & 13,4 & 0 & 0 & 4 \\
\hline Transformación productos hortofrutícolas & 25 & 37,5 & 25 & 0 & 0 & 0 & 12,5 & 0 & 0 & 2,2 \\
\hline Aceite & 26,7 & 33,3 & 6,7 & 13,3 & 13,3 & 0 & 0 & 0 & 6,7 & 4 \\
\hline Cárnicas & 21,5 & 26,2 & 0 & 17,5 & 26,2 & 0 & 0 & 0 & 8,7 & 3,5 \\
\hline Bebidas & 33,3 & 16,7 & 0 & 16,7 & 16,7 & 0 & 0 & 0 & 16,6 & 3,2 \\
\hline Lácteos & 50 & 25 & 12,5 & 0 & 12,5 & 0 & 0 & 0 & 0 & 2,2 \\
\hline Productos metálicos & 40 & 20 & 0 & 20 & 20 & 0 & 0 & 0 & 0 & 2,7 \\
\hline Industria textil & 40 & 40 & 0 & 10 & 10 & 0 & 0 & 0 & 0 & 2,7 \\
\hline Industria de Madera y Mueble & 40 & 30 & 10 & 0 & 10 & 0 & 0 & 0 & 10 & 2,7 \\
\hline Maquinaria mecánica & 44,4 & 22,2 & 0 & 11,1 & 22,3 & 0 & 0 & 0 & 0 & 2,4 \\
\hline Maquinaria eléctrica & 44,4 & 22,2 & 0 & 11,2 & 22,2 & 0 & 0 & 0 & 0 & 2,4 \\
\hline Automóvil & 44,4 & 11,1 & 11,1 & 0 & 11,2 & 0 & 0 & 0 & 22,2 & 2,4 \\
\hline Maquinaria electrónica & 50 & 14,3 & 0 & 7,1 & 28,6 & 0 & 0 & 0 & 0 & 2,2 \\
\hline Productos minerales no metálicos/Industria Química & 57,1 & 14,3 & 0 & 0 & 28,6 & 0 & 0 & 0 & 0 & 1,9 \\
\hline Aeronáutica & 57,1 & 14,3 & 0 & 0 & 28,6 & 0 & 0 & 0 & 0 & 1,9 \\
\hline Industria de Papel & 57,1 & 14,3 & 0 & 14,3 & 14,3 & 0 & 0 & 0 & 0 & 1,9 \\
\hline Industria de Caucho y Plástico & 57,1 & 28,6 & 0 & 14,3 & 0 & 0 & 0 & 0 & 0 & 1,9 \\
\hline Industria de la Confección & 50 & 16,7 & 16,7 & 0 & 16,6 & 0 & 0 & 0 & 0 & 1,6 \\
\hline$\%$ del TOTAL & 31,5 & 31,3 & 3,8 & 9,2 & 12,7 & 0,5 & 3,5 & 1,3 & 6,2 & 100 \\
\hline
\end{tabular}

Fuente: Elaboración propia a partir de nuestras encuestas.

(x) Véase clave en Anexo I

2.- En función del número de proyectos de cooperación tecnológica que los servicios avanzados han desarrollado desde 1997 con otras empresas, la Administración y los organismos públicos de investigación. Para ello la variable número de proyectos se ha cruzado con el tipo de proyecto, localización, sector, tamaño, gastos de innovación y tipo de empresa. Con la información obtenida hemos elaborado varias tablas de doble entrada que nos han servido de base ${ }^{6}$ para conocer cuál es la aportación de los servicios avanzados al proceso de aprendizaje tecnológico de la región y la capacidad de estas empresas para incrementar el nivel tecnológico del espacio competitivo andaluz. 
Es decir, pretendemos estudiar el denominado fenómeno de desparramamiento producido por los servicios avanzados. Su existencia expresa una de las características del modelo interactivo de la innovación, en cuanto que la creación/absorción de conocimientos en las empresas no depende sólo de los esfuerzos individuales sino también de aquéllos a los que tiene fácil acceso. De esta manera la innovación implica actividades de creación colectiva que a menudo se formalizan mediante acuerdos de cooperación entre los elementos del Sistema Regional Innovador (SRI) a través de la cooperación tecnológica, la compra-venta de servicios avanzados, las uniones temporales de empresas, los procesos de spin-off, etc. En este contexto, nos podriamos preguntar también en que fases de la evolución del proceso innovador, según terminología de Porter, se centra la máxima densidad de los flujos (cooperación y contratación de servicios) y qué sectores de la economía andaluza participan en mayor medida.

Del análisis del número de contratos de SA se deduce que en la actualidad la rama que más servicios avanzados demanda en Andalucía es el sector industrial (51,7\%), seguido de los servicios (35\%), la Administración (11,5\%), la agricultura $(3,5 \%$ y la construcción (2,2\%). Por tanto, si la Administración en los años 80 y primeros de los 90 era la promotora y el principal cliente de las empresas de servicios avanzados en Andalucía, hoy ya ocupa un lugar mucho menos importante. Cuestión esta a destacar porque se demuestra que la modernización y la introducción de la innovación en el tejido económico andaluz empieza a ser un hecho constatable. En segundo lugar, conviene subrayar que dentro del sector industrial la actividad agroalimentaria aglutina la mitad de los contratos establecidos, hecho que está en relación con el peso del subsector en la economía andaluza y con la importancia del proceso de modernización que está experimentando.

Pero, como uno de los objetivos más importantes de la investigación era conocer cuál es la aportación de los servicios avanzados al proceso de aprendizaje tecnológico andaluz y la capacidad de estas empresas para incrementar el nivel tecnológico del entorno competitivo, pensamos que debemos detenernos más en estos puntos. En primer lugar conviene recordar que la mayoría de firmas de servicios avanzados (64\%) generan recursos tecnológicos que van asociados a las etapas previas que debe seguir una empresa cuando se quiere incorporar al mundo de la creación de la innovación, según terminología de Porter ${ }^{7}$. Pues,

1. el $53 \%$ de los gastos de innovación que realizan todas estas empresas se dedica a ingeniería, diseño, compra de bienes de equipo, adaptación de tecnología e implantación de innovaciones no tecnológicas.

2. Cuando se efectúa venta de tecnología (50\% de las empresas) es para llevar a cabo asistencias técnicas relacionadas con la ingeniería, diseño, imagen empre- 
sarial, licencias de software y plantas llave en mano. Tecnología que se vende a la industria (42,9\%), a la Administración $(23,8 \%)$, a la construcción $(14,3 \%)$ y a los servicios $(19,2 \%)$. Por lo tanto, desde este punto de vista cabe afirmar que se da un proceso de aprendizaje que se produce a través de las asistencias técnicas, la venta de las licencias de software y la compra-venta de ingeniería, diseño, adaptación de tecnología, etc.

3. Ahora bien, pocas empresas están introducidas en las fases más evolucionadas del proceso innovador, es decir en la realización de I+D (36\%) y muchas menos se hallan posicionadas en el mercado de compra de tecnología propiamente dicha (tan sólo un 15\%), es decir, adquisición de patentes, modelos de utilidad, know how. Por otra parte, aunque hemos señalado ya que la rama económica que más servicios avanzados demanda es la industria, sin embargo la realización de compras de servicios de $\mathrm{I}+\mathrm{D}(1,7 \%)$ y de diseño $(3,8 \%)$ por parte del sector secundario a los SA es reducida. Pensemos que en el entorno andaluz y en este caso en el sector industrial existe una escasa experiencia en el desarrollo del proceso innovador y sobre todo de $\mathrm{I}+\mathrm{D}$ puesto que la mayoría de empresas se iniciaron en la innovación después de 1992 (Figura 1). De ahí que el volumen de gastos que se dedica a la innovación y a la incorporación de infraestructuras innovadoras no da todavía una rentabilidad importante.

Este hecho produce que el grueso $(92,7 \%)$ de las empresas innovadoras que hacen $\mathrm{I}+\mathrm{D}$ y diseño en la industria sean grandes y medianas-grandes pertenecientes a grupos empresariales con un capital de procedencia nacional (22\%), europeo (11\%) y del resto del mundo (6\%). Y, además, esas empresas resuelven los grandes problemas de ingeniería y de $\mathrm{I}+\mathrm{D}$ (químico, material eléctrico y electrónico, agroalimentario, informática, laboratorios de $\mathrm{I}+\mathrm{D}$ etc.) fundamentalmente en colaboración con los OPIs $(64,5 \%)$, sobre todo con las Universidades andaluzas (65\%). No olvidemos que el apoyo de la Administración al desarrollo de I+D lleva a la Universidad a la búsqueda de socios empresariales, lo que refuerza la colaboración OPI-empresa. En ese contexto, dichas colaboraciones les permiten a las empresás reducir inversión en infraestructura y en contratación de técnicos fijos.

Pero también es cierto que estas empresas, ante determinados problemas que no requieren el desarrollo de proyectos, prefieren contratar servicios avanzados, en este caso extranjeros (ingenierías americanas y laboratorios europeos), pues la mayoría de empresas de servicios avanzados andaluces y nacionales no disponen de know-how y de infraestructuras para competir con la Universidad y el CSIC o con firmas de servicios avanzados de carácter multinacional. Por el contrario, cuando las PYMEs industriales hacen I+D, por ejemplo la industria metalmecánica, 
construcción de maquinaria y sector químico, recurren también a la colaboración, pero en este caso con empresas proveedoras del mismo sector, bajo subcontratación, para desarrollar tecnológicamente partes del producto ahorrando costes y aprovechando la experiencia de las firmas proveedoras (Jordá Borrell, Ruiz Rodríguez, Lucendo Monedero, 2001).

Sin embargo, las firmas de servicios avanzados son de tamaño mediano y pequeño $(90,2 \%)$ y, por lo tanto, gastan por término medio un volumen de capital inferior al industrial en innovación. Tanto es así que si comparamos la inversión en I+D entre la industria y los servicios avanzados observamos que se da una proporción de 8 a 1. Por otra parte, la industria está más automatizada y más descentralizada que los SA porque su proceso productivo, además de encontrarse en una fase capitalista más avanzada, se basa en operaciones repetidas, mientras que en los SA este tipo de operaciones son escasas, a excepción de los servicios homologados, y aún así tienen una cierta personalización (gestión de calidad, laboratorios de pruebas y ensayos). En general cabe decir, pues, que los servicios avanzados son personalizados. Frente a la automatización y la maquinaria compleja en la industria, en los SA es muy importante el conocimiento y la cualificación. De ahí que la inversión clave en las empresas de SA no sean los bienes de equipo, que, por otra parte, son relativamente baratos (a excepción de ciertos laboratorios y maquinarias de nuevas tecnologías de la información), sino que la inversión clave es la contratación de recursos humanos, especializados en la economía de la zona, capaces de generar un gran volumen de valor añadido (12,5\%) y de resolver rápidamente los problemas cotidianos o inmediatos de calidad y de innovación de las empresas.

Piénsese que la media de contratación de técnicos superiores y medios por empresa en Andalucía es de 70,4 y si sólo utilizamos los superiores ésta alcanza el índice de 48,5, mientras que en la industria la primera tasa se sitúa en 3,9. Pero, además de estas diferencias estructurales entre la industria y los SA, las empresas localizadas en Andalucía se caracterizan por encontrarse en una fase de desarrollo menos evolucionada que las europeas y americanas.

Por lo tanto, es el sector industrial el que proporcionalmente más gasta en $\mathrm{I}+\mathrm{D}(78,3$ frente a $21,7 \%$ en los $\mathrm{SA})$ y el que más coopera con los organismos públicos de investigación ( 71,5 frente a $28,5 \%$ en los SA). Todo ello dentro de un contexto andaluz en el cual existen proporcionalmente pocas empresas $(36 \%$ en los SA y $42 \%$ del sector industrial) que realicen I+D. Así pues, explicada esa situación de inferioridad innovadora de los SA frente a la industria, podríamos empezar a concluir que los OPIs se presentan como un elemento mucho más importante en la estructuración y jerarquización del medio innovador andaluz que las empresas de servicios avanzados, debido a su liderazgo en el mercado 
de la investigación y a su capacidad para la resolución de problemas a largo plazo, localizándose fundamentalmente en las capitales de provincia más Jerez ${ }^{8}$. En definitiva, desde el punto de vista de la I+D, las OPIs favorecen mucho más el proceso de aprendizaje colectivo que los servicios avanzados, pues debemos pensar que, entre todos los factores explicativos de este proceso de aprendizaje, la $\mathrm{I}+\mathrm{D}$ es el que tiene mayor trascendencia y el que más impacto provoca en el medio innovador andaluz.

\section{Factores innovadores y densidad de redes}

Una vez conocida la aportación de las empresas de servicios avanzados al nivel tecnológico andaluz y al proceso de aprendizaje colectivo desde el punto de vista de la I+D nos parece de interés: a.- profundizar en el comportamiento de los demás factores del proceso innovador (ingeniería, calidad, etc) dentro del mercado de servicios avanzados y b.- analizar la densidad de las redes de la cooperación tecnológica y de los contratos de servicios avanzados.

Pues bien, la compra de servicios avanzados por parte de los sectores productivos andaluces no ha generado hasta ahora una relación muy continua entre ambas partes. Si observamos el número de contratos/empresa/año únicamente el comercio (10) y la industria agroalimentaria $(7,3)$ han demandado frecuentemente servicios. En el resto de sectores, a pesar de que las firmas de servicios avanzados han introducido la búsqueda de vínculos con el cliente (Tablas 1 y 2), la contratación se establece puntualmente: papel (5), productos minerales no metálicos $(2,25)$, material y equipo eléctrico y electrónico $(0,77)$, etc.

Por otra parte, el número de proyectos/empresa colaboradora/año demuestra que este índice es más bajo que el correspondiente a la compra de servicios. Los sectores que más cooperan, como material de transporte $(4)$, informática $(3,6)$, ingeniería $(2,75)$, material de equipo eléctrico y electrónico $(2,5)$, manufacturas diversas $(2,25)$, etc., se sitúan muy por debajo del número medio de contratos efectuados por firma. Firmas que se localizan en el área de influencia de las capitales de Sevilla, Almería, Huelva, Málaga y Jaén más Algeciras y Jerez. En este caso, no siempre son la Universidad y, en menor medida, el CSIC los socios colaboradores de las empresas; en determinadas ocasiones la relación empresa-empresa llega a alcanzar mayor densidad. Pero, en cualquier caso, siempre es mucho mayor la interacción servicioservicio $(46,4 \%)$ o industria-industria $(38 \%)$ que las de tipo mixto servicio-industria $(7,3 \%)$. Por ejemplo, la ingeniería se asocia a menudo con la agroalimentación para mejorar los procesos de fabricación o el sector informático con el de medio ambiente 
para desarrollar software especializado. Igualmente este sector informático coopera con las consultoras para ampliar distribución y venta de software. Pero aun así, de todas estas posibles combinaciones las más intensas y las que alcanzan mayor densidad de relaciones son las de tipo servicio-servicio, bien mediante contratos verbales o bien a través de creación de UTEs o de acuerdos de cooperación, puesto que, como hemos explicado antes, las empresas de SA presentan una gran tendencia a asociarse para complementarse, unas veces para conseguir un crecimiento controlado, otros para trabajar con la administración y en otras ocasiones para desarrollar nuevos servicios.

Según la clasificación sectorial establecida para las empresas de servicios avanzados (Tabla 2), las empresas de gestión de calidad y de consultoría son las que concentran el mayor número de servicios comprados (31,5 y 31,3\% respectivamente), mientras que los demás se encuentran a mucha distancia de ellos. Y es que en Andalucía se está introduciendo de una forma importante la gestión de calidad en las industrias, concertando estos servicios en un $74 \%$ en nuestra región y el resto con empresas españolas. De hecho un 55\% de las empresas industriales y un $45 \%$ de las del sector servicios han introducido la gestión de calidad. De ahí que esas firmas hayan implantado mejoras organizativas y cambios operativos. En consecuencia, dado que la gestión de calidad implica formación, asesoramiento y reorganización de la empresa, podemos decir que la gestión de calidad y la consultoría están estrechamente relacionadas. Esto es, ambas funciones de SA son las que producen la mayor difusión del conocimiento a los demás sectores económicos andaluces y las que más contribuyen al proceso de aprendizaje colectivo. Lo que ocurre es que estas funciones se sitúan en las fases anteriores a la etapa de innovación propiamente dicha, pues cuando se estudia la estructura económica andaluza se deduce que se encuentra en la fase de los factores avanzados según terminología de Porter (1991), ya que los sectores más importantes se basan en los recursos naturales (agrario, agroalimentario, minero...) y/o compiten en términos de precios. Por otra parte, estas actividades (agroalimentario, comercio...) son generalmente de bienes de consumo final y no son grandes demandadoras de servicios avanzados.

Sin embargo, la demanda de ingeniería por parte de los sectores productivos alcanza tan sólo un 12,7\% (Tabla 2). Éste es un valor ciertamente bajo, (salvo en la industria de maquinaria, aeronáutica y productos metálicos, donde la producción de planos es fundamental y los porcentajes de compra suben por encima de la media) y la explicación radica en que el proceso innovador de las empresas andaluzas se ha basado hasta ahora en la compra masiva de bienes de equipo (36,2\% sobre el total de compra de tecnología), compra que se viene realizando desde comienzos de los años ochenta, especialmente de sistemas de organización flexible y de gestión y distribución de almacén; sin embargo, la asistencia técnica o la compra de plantas llave en mano únicamente representa el 21,2 y el 19\%. 
Tabla 3. Cooperación con las OPIs por tipo de proyectos y sectores (\%)

\begin{tabular}{|c|c|c|c|c|c|c|c|c|c|c|c|c|c|c|c|c|c|}
\hline \multirow[b]{2}{*}{ Tipo de proyecto } & \multicolumn{17}{|c|}{ Sector destinatario $(x)$} \\
\hline & $A$ & $D A$ & $D E$ & $D G$ & $D I$ & DJ & $D K$ & $D L$ & $D M$ & $D N$ & $G$ & $I N D$ & Cal & Inf & & $\begin{array}{c}L a b o r \\
1+D\end{array}$ & SER Tolal \\
\hline Desarrollo de mercado & 0 & 1.9 & 0 & 0 & 0 & 0 & 0 & 0 & 0 & 0 & 0 & 1,9 & 0 & 2,8 & 1,4 & 0 & $4,2 \quad 6,1$ \\
\hline Formación & 0 & 0 & 0 & 0 & 0 & 0 & 0 & 0 & 0 & 0 & 0 & 0 & 0 & 1,8 & 0 & 0 & $1,8 \quad 1,8$ \\
\hline Gestión de la calidad & 0 & 0 & 0 & 0 & 0 & 0 & 0 & 0 & 0 & 0 & 0 & 0 & 1,5 & 0 & 0 & 0 & $1,5 \quad 1,5$ \\
\hline$I+D$ & 5,7 & 5,7 & 0 & 10,2 & 4,3 & 5,7 & 2,9 & 10 & 0 & 1,4 & 1,4 & 47,3 & 1,4 & 7,1 & 4,3 & 4,3 & $17,1 \quad 64,4$ \\
\hline I+D e Ingeniería & 0 & 0 & 0 & 1,4 & 0 & 0 & 0 & 0 & 0 & 1,4 & 0 & 2,8 & 0 & 0 & 0 & 0 & 2,8 \\
\hline Ingeniería & 0 & 1,4 & 0 & 1,4 & 1,4 & 2,9 & 1,4 & 1,4 & 0 & 1,5 & 0 & 11,4 & 0 & 2,0 & 0 & 0 & $2,0 \quad 13,4$ \\
\hline Intercambio material para la investigación & 1,4 & 0 & 0 & 0 & 0 & 0 & 0 & 0 & 0 & 0 & 0 & 1,4 & 0 & 0 & 0 & 0 & 1,4 \\
\hline Diseño & 0 & 0 & 0 & 0 & 1,4 & 0 & 0 & 1,4 & 2,9 & 0 & 0 & 5,8 & 0 & 1,4 & 0 & 0 & $1,4 \quad 7,2$ \\
\hline Encuestas & 0 & 0 & 1,4 & 0 & 0 & 0 & 0 & 0 & 0 & 0 & 0 & 1,4 & 0 & 0 & 0 & 0 & $0 \quad 1,4$ \\
\hline TOTAL & 7,1 & 9.0 & 1,4 & 13 & 7,1 & 8,6 & 4,3 & 12,9 & 2,9 & 4,3 & 1,4 & 72,0 & 2,9 & 15,1 & 5,7 & 4,3 & $28,0 \quad 100$ \\
\hline
\end{tabular}

Fuente: Elaboración propia a partir de nuestras encuestas.

(x) Véase clave en Anexo I

Por lo tanto, las empresas demandantes de servicios de ingeniería, fundamentalmente la industria, aunque también es importante la demanda del sector servicios (Tablas 1 y 2), suelen contratarla bien con la Universidad (Tabla 3), bien dentro del propio sector secundario o bien la compran a las firmas de servicios avanzados al objeto de reorganizar el proceso productivo adaptando los nuevos equipos a sus necesidades, contratando a empresas informáticas y de formación (por falta de especialistas) e introduciendo innovaciones no tecnológicas de tipo organizativo. Así pues, aunque la ingeniería se compre fundamentalmente en el mercado regional, las firmas de SA no ostentan un dominio y ni mucho menos un liderazgo en el ámbito andaluz en cuestiones de ingeniería, a pesar de que sus gastos de innovación son relevantes $(11,4 \%)$ dentro del propio sector. En segundo lugar, el porcentaje de trabajo de ingeniería demandado nos está indicando una vez más que el proceso de modernización de la economía andaluza se encuentra en la fase anterior a la de innovación propiamente dicha, según terminología de Porter (Jordá Borrell, 1998).

De la misma manera, existen otros subsectores, por ejemplo todos los servicios relacionados con la informática (software, implantación de sistemas informáticos, telemáticos, internet, etc), que están contribuyendo enormemente a modernizar los sectores productivos andaluces. Si la demanda es reducida ( $17,4 \%$ para la industria y $9,2 \%$ para el conjunto de ramas económicas, como indican las tablas 1 y 2) frente a la adquisición de servicios de gestión de calidad, es porque cada vez más el software está estandarizado, saliendo al mercado aplicaciones específicas para cada subsector económico, incluso existen empresas especializadas en la venta de programas para la gestión de grandes empresas y grupos empresariales. La cuestión estriba entonces en si estas firmas andaluzas de informática tienen capacidad de liderazgo para imponerse en mercados no regionales, teniendo 
en cuenta que invierten en innovación el 38,1\%, es decir, más que ningún otro subsector de SA.

Pues bien, estas empresas hacen desarrollos tecnológicos o ingeniería de software, cuya misión es investigar técnicas avanzadas de gestión informática para ofrecer soluciones integradas concretas. En consecuencia, cabe deducir que estas empresas suministran a sus clientes un servicio postventa muy válido y unos productos nuevos o mejorados a escala regional y en las provincias españolas más próximas a Andalucía. Pero, a pesar de registrar sus productos en la oficina nacional de patentes y estar muy especializados en segmentos de mercado muy pequeños, no son capaces de imponer su liderazgo a escala regional y/o nacional porque les falta tecnología para competir con las grandes marcas, máxime teniendo en cuenta que el ciclo productivo del software es muy corto y las firmas andaluzas se tienen que adaptar al ritmo de innovación de las grandes multinacionales.

Ahora bien, también cabría pensar que la demanda de servicios avanzados que no corresponde a la gestión de calidad y a la consultoría es baja porque se contratan fuera de Andalucía. La información recogida a través de 150 encuestas correspondientes a los sectores industrial y de servicios avanzados nos demuestra (Tabla 1) que los servicios que no son comprados en Andalucía son los que van ligados con los procesos de globalización (asesoramiento comercial y logístico, estudios de mercado, comercio exterior, costes y estudios de viabilidad, etc) y los de I+D y diseño industrial. Contratos que son establecidos sobre todo por los sectores de la industria de la alimentación, química, construcción de maquinaria y material de transporte. Es decir, los sectores que están internacionalizados desde el punto de vista de los clientes y de los proveedores y en los que predomina la mediana y la gran empresa, aunque un porcentaje importante de compra de servicios se realiza en Andalucía, por ejemplo, publicidad, control y planificación, gestión de calidad, etc.

En el terreno de la cooperación tecnológica, la escala internacional (Tabla 4) destaca cuando las firmas pertenecen a grupos empresariales (la agroalimentación, metalurgia, química y material eléctrico y electrónico, la ingeniería, la informática y gestión de calidad) puesto que son éstos los que poseen know how e infraestructuras suficientes para participar en proyectos internacionales. Ahora bien, tiene mayor importancia la colaboración en el seno del mismo grupo (23,8\% en la industria y $6,8 \%$ en los servicios) porque a la hora de hacer transferencia de tecnología prefieren hacerla entre matriz y filial o entre filial y filial, aprovechando su especialización funcional. Sin embargo, la cooperación de estos grupos de empresas con los Organismos Públicos de Investigación ( $9,6 \%$ en la industria y $2 \%$ en los servicios avanzados) es escasa, a no ser que tengan posibilidades de acaparar los resultados comerciales de la investigación. 
Tabla 4. Cooperación con OPIs por localización de OPI y sectores (\%)

\begin{tabular}{|ccccccccc|}
\hline \multicolumn{7}{c}{} & \multicolumn{7}{c|}{ Ambito } & & \\
Secior $(x)$ & REGIONAL & REG-NAC & REG-NAC-UE & NACIONAL & NAC-EU & EUROPA & Total \\
\hline A & 6,3 & 0 & 1,6 & 0 & 0 & 0 & 7,8 \\
DA & 7,8 & 1,6 & 0 & 0 & 0 & 0 & 9,4 \\
DE & 1,6 & 0 & 0 & 0 & 0 & 0 & 1,6 \\
DG & 9,4 & 1,6 & 1,6 & 1,6 & 0 & 0 & 14,1 \\
DI & 1,6 & 0 & 0 & 0 & 0 & 0 & 1,6 \\
DJ & 9,4 & 0 & 0 & 0 & 0 & 0 & 9,4 \\
DK & 1,6 & 0 & 0 & 3,1 & 0 & 0 & 4,7 \\
DL & 14,1 & 0 & 1,6 & 0 & 1,6 & 0 & 17,2 \\
DM & 1,6 & 0 & 0 & 3,1 & 0 & 0 & 4,7 \\
DN & 3,1 & 0 & 0 & 3,1 & 0 & 0 & 6,3 \\
G & 1,6 & 0 & 0 & 0 & 0 & 0 & 1,6 \\
IND & 58,1 & 3,2 & 4,8 & 10,9 & 1,6 & 0 & 78,4 \\
Cal & 1,6 & 0 & 0 & 0 & 0 & 1,6 & 3,1 \\
Infor & 12,5 & 0 & 0 & 0 & 0 & 0 & 12,5 \\
Ing & 4,7 & 0 & 0 & 1,6 & 0 & 0 & 6,3 \\
SER & 18,8 & 0 & 0 & 1,6 & 0 & 1,6 & 21,9 \\
\hline TOTAL & 76,6 & 3,1 & 4,7 & 12,5 & 1,6 & 1,6 & 100 \\
\hline
\end{tabular}

Fuente: Elaboración propia a partir de nuestras encuestas.

(x) Véase clave en Anexo I

\section{Conclusiones}

En definitiva, el grueso de la contratación de servicios y de la cooperación (intrasectorial y entre sectores) se realiza a escala regional ( $63 \%$ y $76,6 \%$ respectivamente, como se puede ver en las tablas 1 y 4 ), bien con OPIs para desarrollar $\mathrm{I}+\mathrm{D}$ e Ingeniería (15\%), bien con clientes industriales (35\%), bien con competidores. De esta situación se derivan aspectos positivos y otros que requieren mejorarse. En el primer caso, conviene destacar que la universidad' y los SA (Figura 2) han generado recursos endógenos y un mercado local/regional de servicios importante en un corto espacio de tiempo, a través de la constitución de redes verticales y horizontales. Pero este mercado regional no forma un continuo espacial resultado de la magnitud de los flujos y del desarrollo de la sociedad servindustrial, sino que está constituido por diversos espacios-isla (mercados locales/comarcales) escasamente conectados entre sí, debido a que las empresas existentes de SA no tienen capacidad para crear un mercado propiamente regional, sino a escalas más reducidas. Estos espacios-isla están representados por las capitales de provincia y sus áreas de influencia que es doncle se concentran los flujos de oferta-demanda de SA por parte de la industria (Figura 2) y de la administración, mientras las ciudades medias de carácter industrial todavía no han desarrollado mercados de oferta debido al bajo nivel innovador de su proceso de industrialización. 
Las causas de esta ordenación vendrían explicadas por la distribución de la actividad económica, en este caso la industria, por la localización de los centros de decisión de la administración, por el nivel de los procesos de industrialización y de innovación y por el tipo de sectores predominantes. Por otra parte, en la estructura de esa distribución aparece una jerarquía urbana fundamentada en la magnitud de cada uno de los mercados comarcales/provinciales (áreas de influencia de las capitales) que han generado los flujos oferta-demanda y el número de niveles (local, regional, nacional, internacional) en que están organizados éstos en cada núcleo. En este contexto, convendría exponer también los factores explicativos del desarrollo de estos mercados comarcales/provinciales, los cuales serían el volumen de establecimientos industriales y de los centros de la administración localizados en los núcleos, el tipo de sector, el tamaño de las empresas y el nivel innovador de sus procesos. Así, el $80 \%$ de las sinergias se concentran en el conjunto de las áreas de influencia de Sevilla, Almería Huelva y Jaén (Figura 2). Por último, en esa ordenación jerárquica, Sevilla ocupa el primer lugar debido a que es la capital regional y una ciudad de rango nacional, influyendo ambos elementos en el grado de concentración de los flujos y en su capacidad de atracción de aquellas empresas que buscan un estatus y una imagen. Estos factores constituyen un indicador del importante papel que juega Sevilla como exportadora de SA a las otras capitales, a Jerez y a Algeciras.

Aunque no disponemos de datos de flujos (compraventa de SA y/o cooperación) ni de información sobre la conformación de los mercados de SA en otras comunidades autónomas, sí podemos comparar a grandes rasgos la estructura andaluza de localización de los SA con la existente en algunos ámbitos regionales. De las investigaciones realizadas hasta ahora observamos que las regiones periféricas, por ejemplo Castilla León, siguen unas pautas de localización (Rubalcaba y otros, 1998; Sánchez Tomé, 1997) muy semejantes a las de Andalucía. Sin embargo, las comunidades autónomas que han alcanzado un proceso de industrialización avanzado cuentan con una distribución intrarregional de oferta-demanda configurada por muchos más polos que las periféricas y al mismo tiempo éstos están organizados de forma complementaria y funcional debido a la difusión del desarrollo local endógeno y al mayor tamaño de las empresas. Así en Euskadi tan sólo un $59 \%$ de los establecimientos (Rubalcaba y otros, 1998) se concentran en las tres capitales vascas, el resto se ubica en las zonas industriales (núcleos de tipo medio). En la Comunidad Valenciana, Valencia aglutina el $49 \%$ de los SA mientras que juegan un papel importante ciertos centros de tamaño intermedio (Salom y otros, 1999). Sin embargo, Cataluña que disfruta de un nivel de industrialización de características semejantes a las del País Vasco se diferencia de las comunidades autónomas anteriores porque Barcelona, aparte de haber desarrollado una macrocefalia relevante, posee rango internacional y las empresas buscan en su localiza- 
ción aspectos ligados al estatus o a la imagen. Estos hechos justifican que Barcelona englobe el $65 \%$ de las firmas catalanas de SA y en el área metropolitana, donde existe un número considerable de grandes núcleos intermedios, se concentre el 93\% del total (Soy, 1997).

Por otra parte, y de acuerdo con el nivel de información de que disponemos sobre Andalucía, cabe inferir que en los mercados locales/comarcales/provinciales están presentes al menos dos tipos de flujos:

1. unos ligados a las funciones de proximidad y polaridad debido al carácter personalizado de estos servicios y a la poca capacidad de las empresas de SA de desarrollar un mercado propiamente regional. Ahora bien, dado que nos interesaba conocer el nivel tecnológico de estos espacios-isla (mercados locales/ comarcales/ provinciales) deducimos de los resultados de la investigación (Tabla 4) que un porcentaje elevado de los flujos de proximidad $(76 \%)$ estân constituidos por redes bilaterales o simples que son mucho menos eficaces que las complejas a la hora de difundir innovaciones y generar desparramamientos. Además, tal como se ha demostrado en el trabajo, el consumo de SA en los procesos productivos no es muy elevado debido a que la industria se encuentra en la fase de mejora de la calidad o, lo que es lo mismo, no está todavía muy terciarizada. De ahí que el tipo de servicios que se adquieren en mayor medida son los de gestión de calidad y los de consultoría.

2. El otro tipo de flujo (Figura 2) desarrollado en los mercados locales/ comarcales está vinculado a la noción de vecinaje tecnológico y tiene que ver con la homogenización del nivel tecnológico en la distancia (por ejemplo en Sevilla, Huelva y Jaén). Estas relaciones aparecen expresadas bien bajo la forma de cooperación, compra-venta, procesos de spin-off, uniones temporales de empresas, etc; bien a través de sinergias de importación-exportación de SA. Por ello, si nos preguntamos para qué han desarrollado este tipo de flujos las empresas localizadas en Andalucía concluiríamos que:

a) para introducirse en otros mercados, por ejemplo, incrementando el nivel de exportación a la U.E. y a USA (25\%), para internacionalizarse en países subdesarrollados $(32 \%)$ y para establecerse en otras comunidades autônomas (43\%). En consecuencia, podemos afirmar que el crecimiento de este tipo de flujos ha sido beneficioso para aumentar la capacidad de apertura al exterior de Andalucía. 
b) para realizar $I+D$, introducir innovaciones e incrementar el proceso de aprendizaje a través de la formación de redes complejas. Sin embargo, estas agrupaciones/redes con enlaces multisectoriales y organizaciones de diversos perfiles ( $14 \%$ del total) llevan a cabo escasa transferencia tecnológica (Tabla 4) por la falta de integración vertical de los sectores y por el bajo nivel tecnológico de las empresas andaluzas, pues éstas utilizan insumos con poca diferenciación y componentes básicos, lo que impide vender sus productos en el mercado regional al reducido número de firmas que hacen $I+D$.

En este contexto, el proceso de acumulación de conocimientos es pobre, poco complejo y con un nivel tecnológico reducido. Todo ello enmarcado en un escenario empresarial en el que la innovación se define como una manera de solucionar problemas muchas veces de costes, en un escenario en que la innovación parte de una idea y no de la investigación básica o aplicada, en un escenario en que los empresarios de los servicios avanzados e industriales innovan para mejorar la calidad y no para convertirse en líderes europeos o en el mercado internacional. Por consiguiente, los servicios avanzados solamente pueden constituir un elemento clave para la renovación del sistema productivo, para la adaptación de tecnología y para mejorar los productos pero difícilmente pueden serlo para la creación de nuevas tecnologías y para introducir la economía del conocimiento.

\section{Notas}

1 Se pueden proponer como actividades avanzadas los servicios incluidos en la categoría $\mathrm{K}$ de la Clasificación Industrial Estándar de las Actividades Económicas (ISIC), que engloba a las empresas de publicidad, informáticas, ingenierías, consultoras, empresas de normalización y control, laboratorios de $\mathrm{I}+\mathrm{D}$ y de pruebas y ensayos, auditorías, medio ambiente y gestión de calidad (Commission European Communities, 1999).

2 Según nuestras investigaciones la implantación de sistemas de organización flexible en la industria representó un $15 \%$ entre $1985-89$, un $38,6 \%$ entre 1989-94 y un 36,4\% entre 1995-1999 (Jordá, Ruiz, Lucendo, 2001).

3 La dimensión media de las empresas de SA con sede central en Andalucía es inferior al promedio del conjunto de firmas establecidas en la Comunidad Autónoma. Así, el 68\% de las firmas con sede en Andalucía tienen entre 1 y 10 empleados, porcentaje que se reduce 10 puntos si se considera el conjunto de ellas catalogadas en nuestro Directorio de 2000.

4 Las innovaciones radicales suponen una rotura súbita respecto al estado anterior de la tecnología, dando como resultado productos y procesos totalmente nuevos mientras que las incrementales se definen como mejoras de los productos y procesos ya conocidos; sin embargo, una innovación es imitativa cuando se copian los productos/servicios de la competencia sin añadirle ninguna característica que les diferencie. 
5 El Manual de Frascati define las actividades de I+D como el conjunto de actividades creativas de forma sistemática a fin de aumentar el caudal de conocimientos científicos, técnicos y utilizarlos para introducir nuevas aplicaciones

6 Dichas tablas no las hemos podido incorporar a este trabajo por motivos de espacio.

7 Este autor distingue cuatro etapas perfectamente diferenciadas para representar a muy grandes rasgos los problemas característicos a que se enfrentan las empresas de los países/regiones, en diferentes momentos, así como las fuerzas que impulsan el progreso o la decadencia de su economía. De esta forma tendríamos: la impulsada por el desarrollo de los factores (básicos y avanzados); la impulsada por la inversión; la impulsada por la innovación; y la impulsada por la riqueza.

8 Nos hemos centrado en los sectores industrial y de servicios avanzados a la hora de analizar la importancia de la generación de I+D porque son prácticamente los dos únicas ramas que efectúan investigación más desarrollo.

9 La única área metropolitana que ha iniciado el proceso de descentralización de servicios es Sevilla y en ese sentido cabe mencionar la localización de dos universidades (ademâs de la de Sevilla) emplazadas una en Dos Hermanas y la otra en Bormujos, aunque prácticamente están empezando ahora a desarrollar sus funciones.

\section{Bibliografía}

Antonelli, A. (1999): Localized technological change, new information technology and the knowledge-based economy: the European evidence. Joumal of Evolutionary Economics, 8(2), p. 177-198.

Ardán (2001): Directorio e informe económico financiero de 10.000 empresas de Andalucia. Vigo, IFA, Analistas económicos de Andalucía.

Buesa, M. y Molero, J. (1992): Patrones del cambio tecnológico y politica industrial. Madrid, Civitas. •

Cuadrado Roura, J. y Rubalcaba Bermejo, L. (2000): Los servicios a empresas en la industria española. Madrid, Instituto de Estudios Económicos.

Camagni, R. (1991): Innovation Network, Spatial perspectives. Londres, Belhaven press.
Commission European Communities (1999): The Contribution of Business Services to Industrial Performance. Luxemburgo, DGIII.

European Commission (2001): The contribution of bussiness services to industrial performance. A common policy framework. Luxemburgo, DGIII industry.

Fache, J. (2002): Les territoires de la baute technologie. Rennes, Presses Universitaires de Rennes.

IFA (1993): Catálogo de empresas de servicios avanzados de Andalucia (SERAVAN). Sevilla, Instituto de Fomento Andaluz.

IFA (1997): Catálogo de empresas de servicios avanzados en Andalucia (SERAVAN). Sevilla, Instituto de Fomento Andaluz.

Jordá Borrell, R. (1995): PME, régions intermédiaires et globalisation. En 
Julien, P. Leo, G. y Philippe, Ph.: PME et grands marchés. Paris, Harmattan, $\mathrm{p}$. 147-162

Jordá Borrell, R. (1997): Nuevas tecnologías. En Vallés (Coord): Economía Española. Madrid, Mc Graw-Hill, p. 643-664.

Jordá Borrell, R. (1998): Formación del sistema ciencia-tecnología-industria. En Vallés (Coord): Economia Andaluza. Sevilla, Algaida editorial.

Jordá Borrell, R., Ruiz Rodriguez, F. y Lucendo Monedero, A. (2001): El entorno productivo y su incidencia en el desarrollo del proceso innovador de las empresas andaluzas. Sevilla, Instituto de Estadística Andaluz.

Jordá Borrell, R. (2002): Servicios a empresas (SEMP) y territorio. En López Lara, Miranda Bonilla y Posada Simeón (Ed): Los servicios y los transportes en las transformaciones territoriales. Asociación de Geógrafos Española, Universidad de Sevilla (en prensa).

Kline, J. y Rosenberg, N. (1986): An overview of innovation. Washington, The National Academy Press.

Marquis, D. (1969): The anatomy of succesful innovatios. Innovations, 1(7), p. 32-45.

Miles, I. (1995): Innovation in Services. Part 2: Sectorial and Industrial Studies of Innovation. En $M$ Dodgson $y \quad R$. Rothwell (Eds.): The Handbook of Industrial Innovation. Londres, Edwar Elgar, p. 243-256.

Nelson, R. y Winter, S. (1984): An evolutionary theory of economic change. Cambridge, Harvard University Press.
OCDE (1994): Frascati Manual, main definitions and conventions for the measurement of researcb and experimental development (RED). Paris, OCDE.

Ochel, W. y Wegner, M. (1987): Service economies in Europe: opportunities for growth. Londres, Printer Pub \& Wesriew Press.

Porter, M. (1991): The competitive advantage of nations. Londres, MacMillan.

Rubalcaba Bermejo, L. et al. (1998): Crecimiento y geografia de los servicios a empresas en el contexto de la nueva sociedad servindustrial: el caso de la Comunidad de Madrid. Madrid, Instituto de Estadística de la Comunidad de Madrid.

Ruyssen, O. (1987): The new deal in services: a challege for Europe, en Akeehurst y Gadrey (Eds.): The economics of services. Londres, Frank Cass.

Salom, J., Albertos, J., Pitarch, D. y Delios, E. (1999): Sistema urbano e innovación industrial en el Pais Valenciano. Valencia, Departamento de Geografía, Universidad de Valencia.

Sánchez Tomé, E. (1997): Servicios a las empresas. En Los servicios a la producción y el desarrollo regional en Castilla León, III Jornadas CastellanoLeonesas de Ciencia Regional. Salamanca, Junta de Castilla León y Asociación Castellano Leonesa de Ciencia Regional, p. 109-114.

Soy, A. (1997): Servicios a las empresas y desarrollo regional y urbano: el caso de 
Cataluña. Economía Industrial, 313, p. 105-114.

Utterback, J. (1971): The process of technological innovations within the firm.
Academy of Management Journal, 18, p. 3-19.

Veltz, P. (1999): Mundialización, ciudades $y$ territorio. Barcelona, Ariel. 


\section{ANEXO I}

\section{ABREVIATURAS DE LAS TABLAS}

\section{Denominación de los sectores}

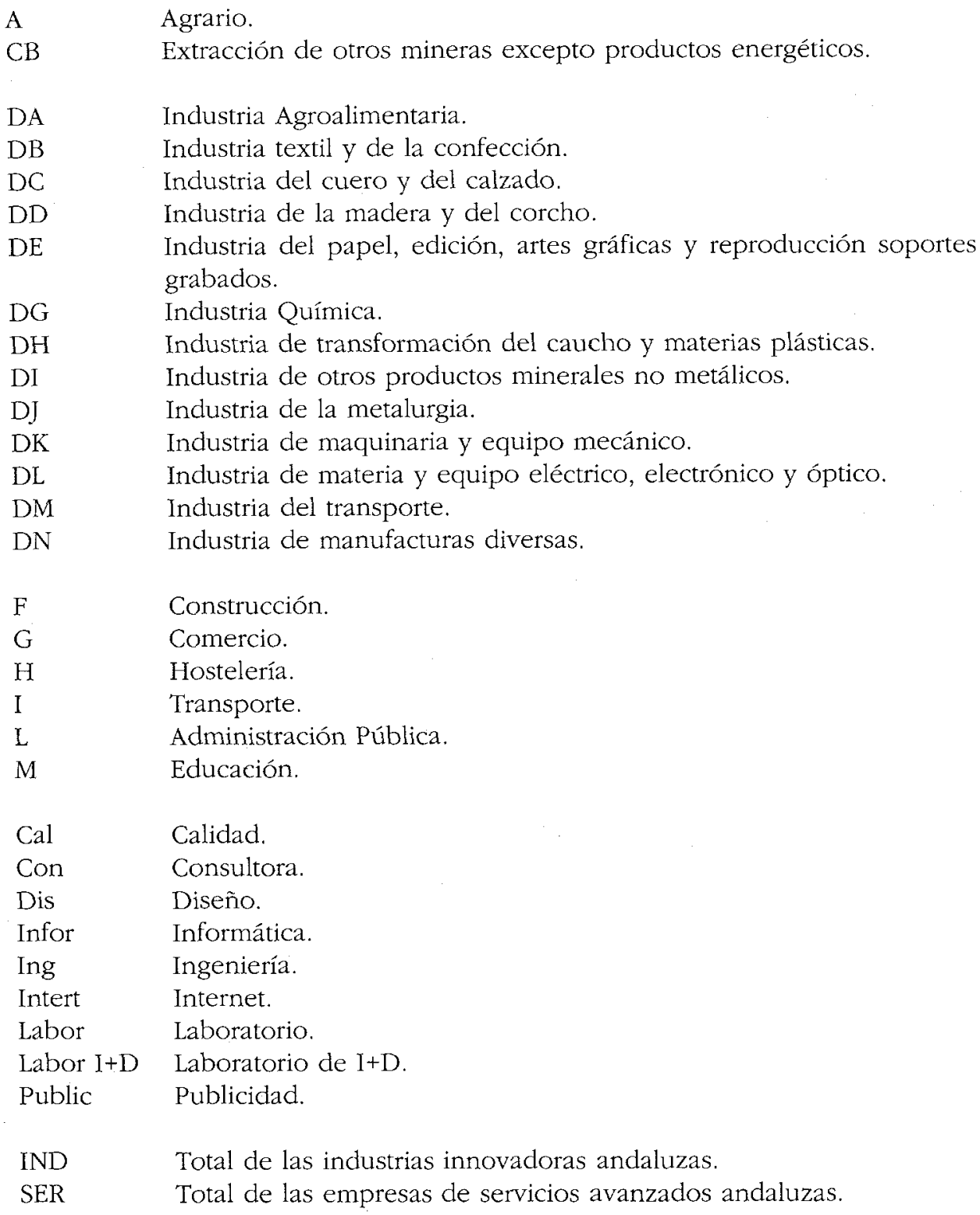

IND Total de las industrias innovadoras andaluzas.

SER Total de las empresas de servicios avanzados andaluzas. 